Revue internationale P.M.E.

Économie et gestion de la petite et moyenne entreprise

\title{
Problématique et particularités des nouvelles entreprises. Le cas d'Alicante
}

\author{
Enrique Claver Cortés, José María Gómez, Juan Lopis et José Luis Gascó
}

Volume 6, numéro 3-4, 1994

URI : https://id.erudit.org/iderudit/1008234ar

DOI : https://doi.org/10.7202/1008234ar

Aller au sommaire du numéro

\section{Éditeur(s)}

Presses de l’Université du Québec

ISSN

0776-5436 (imprimé)

1918-9699 (numérique)

Découvrir la revue

Citer cette note

Cortés, E. C., Gómez, J. M., Lopis, J. \& Gascó, J. L. (1994). Problématique et particularités des nouvelles entreprises. Le cas d'Alicante. Revue internationale P.M.E., 6(3-4), 131-144. https://doi.org/10.7202/1008234ar
Résumé de l'article

Cet article analyse les caractéristiques des nouvelles petites entreprises dans la province d'Alicante, en Espagne, notamment durant les années 80. À partir d'un questionnaire soumis aux nouveaux propriétaires-dirigeants, nous avons voulu connaître ces entreprises et les caractéristiques de leur dirigeant. Notre enquête s'est arrêtée d'abord aux profils personnels prédominants distinguant ceux-ci de la population, et ensuite aux motifs expliquant leur action. Ces motifs proviennent tant de leurs aspirations personnelles et professionnelles que des besoins et des opportunités économiques. Une fois lancées, les nouvelles entreprises suivent une voie propre avec tout ce que cela entraîne et font face à des problèmes concrets que nous essayons de définir dans cette étude.
Tous droits réservés @ Presses de l’Université du Québec, 1994
Ce document est protégé par la loi sur le droit d'auteur. L'utilisation des services d’Érudit (y compris la reproduction) est assujettie à sa politique d'utilisation que vous pouvez consulter en ligne.

https://apropos.erudit.org/fr/usagers/politique-dutilisation/ 


\title{
Problématique et particularités des nouvelles entreprises. Le cas d'Alicante
}

\author{
Dr. Enrique Claver CORTÉS* \\ Dr. José María Gómez GRÁS** \\ Dr. Juan Llopis TAVERNER*** \\ José Luís Gascó GASCÓ****
}

\begin{abstract}
RÉSUMÉ
Cet article analyse les caractéristiques des nouvelles petites entreprises dans la province d'Alicante, en Espagne, notamment durant les années 80 . $\dot{A}$ partir d'un questionnaire soumis aux nouveaux propriétaires-dirigeants, nous avons voulu connaître ces entreprises et les caractéristiques de leur dirigeant. Notre enquête s'est arrêtée d'abord aux profils personnels prédominants distinguant ceux-ci de la population, et ensuite aux motifs expliquant leur action. Ces motifs proviennent tant de leurs aspirations personnelles et professionnelles que des besoins et des opportunités économiques. Une fois lancées, les
\end{abstract}

* Prof. Dr. Enrique Claver Cortés est Catedrático de Organización de Empresas de l'Université d'Alicante. Il est directeur et coordinateur de plusieurs projets de recherche sur la création d'entreprises et les ressources humaines.

** Dr. José María Gómez Grás est professeur titulaire de Organización de Empresas de l'Université d'Alicante. Il travaille sur la création d'entreprises, le capital de risque et les subventions aux entreprises.

*** Dr. Juan Llopis Taverner est professeur titulaire de Organización de Empresas de l'Université d'Alicante. Il a fait des recherches sur la création d'entreprises en plus des études sectorielles sur les entreprises. Il étudie aussi la culture d'entreprise.

**** José Luís Gascó Gascó est professeur titulaire à l'Escuela Universitaria de l'Université d'Alicante. Ses intérêts de recherche visent la création d'entreprise et la gestion des ressources humaines.

Adresse : Departamento de Organización de Empresas, Universidad de Alicante, Ctra. de San Vicente del Raspeig s/n, 03690 San Vicente (Alicante), ESPAÑA. 
nouvelles entreprises suivent une voie propre avec tout ce que cela entraîne et font face à des problèmes concrets que nous essayons de définir dans cette étude.

\section{ABSTRACT}

This article presents an analysis of the behavior characteristics of new small businesses in the province of Alicante, Spain, during the 1980's. A questionnaire was sent to all new business owner operators which provided information about both the business and the owner operator. Our study was concerned with the identification of individual personal owner characteristics which distinguish entrepreneurs from the general population as well as the factors which motivate them. These personal motives derive from both personal and professional aspirations as well as need and economic opportunity. Once started, new businesses continue to evolve and forge their own way with all that this entails. In this study we attempt to describe how these new businesses respond to real problems.

\section{RESUMEN}

Este artículo analiza las caractérísticas de las pequenas empresas de nueva creación en la provincia de Alicante (España), notadamente durante la decada de los años 80. A partir de un cuestionario dirigido a los nuevos proprietáriosdirigentes (promotores), hemos buscado comocev estas empresas y los caracteristicas de sus regentes. Nuestra investigacion primero personales predominantes que pudiesen delimitor a estos emprendedoves del total de la población potencial. Después, estos promotores tuvieron unas ideas más o menos reflexionadas antes de adquirir esta condición y que podrían estar relacionadas con ciertos estímulos como las aspiraciones personales $y$ profesionales, las posibles necesidades económicas o la facilidad de acceso. Una vez que estas personas acceden, por cualquier conducto, a tener la naturaleza referenciada de nuevo empresario, crean una unidad económica que va a tener vida propia con todo lo que ello entraña. De este modo, las firmas recientes poseen un tamaño determinado, normalmente pequeño, con unos problemas concretos y unas características definidoras de las mismas, que intentamos delimitar en este trabajo.

\section{Introduction}

Lorsqu'une personne ou un groupe d'entrepreneurs décident de mettre en pratique leurs idées pour créer une entreprise, ils mettent sur pied une entité juridique et économique selon des modalités qui ne seront pas nécessairement les mêmes quand l'entreprise sera mature. Cette entreprise aura sa propre vie avec tout ce que cela implique. Ses caractéristiques et son développement seront fonction du type de produits offerts, des clients visés, des technologies utilisées, du financement afférent et des relations avec l'environnement. Tout ceci peut influencer les comportements du chef d'entreprise. 
On a presque toujours soutenu que pour créer des entreprises, il fallait qu'il existe dans l'environnement des facteurs particulièrement favorables à l'initiative entrepreneuriale. L'hypothèse que nous utilisons ici est que les facteurs décisifs pour la création d'entreprise sont, d'une part, cet environnement socioéconomique favorable ou défavorable, mais aussi, d'autre part, une forte motivation des entrepreneurs potentiels. Parmi ces motifs, on peut souligner la recherche d'indépendance personnelle, la mise en pratique d'idées originales, la capacité de diriger une organisation, une certaine indépendance économique, une capacité de travail élevée, la recherche d'un revenu plus avantageux, le besoin de développer un patrimoine personnel, etc.

Les difficultés que l'entrepreneur doit surmonter touchent le financement de départ, l'environnement économique et fiscal, l'image du chef d'entreprise dans le milieu, sa forınation comme gestionnaire potentiel, le risque personnel, les entraves bureaucratiques et légales, etc. Ces obstacles font que le nombre net de nouveaux entrepreneurs est plus élevé dans certaines économies que dans d'autres, notamment dans les pays développés.

En tenant compte de ces variables, notre recherche a porté sur une région géographique méditerranéenne, la province d'Alicante, qui, depuis les dix dernières années, est reconnue pour avoir une économie à expansion croissante.

\section{Les caractéristiques des nouvelles entreprises}

Une grande partie de la documentation sur la petite entreprise attache une grande importance aux caractéristiques et aux problèmes de celle-ci. Cependant, il n'existe pas beaucoup d'évidences empiriques sur les classifications des entreprises créées récemment, ni d'éléments qui permettent de bien distinguer les différentes problématiques. Traditionnellement, les recherches qui ont marqué ce domaine ${ }^{1}$ se sont basées sur des hypothèses permettant de définir un modèle de création d'entreprise. Dans ces recherches, les entreprises seraient soumises à l'influence personnelle du chef-propriétaire. Ce serait le plus souvent de petites unités avec peu d'employés, un chiffre d'affaires et un impact sur le marché peu important, un caractère local au plan de la gestion, un degré d'autonomie décisionnelle très bas, une structure organisationnelle simple, conservatrice et peu innovatrice tant en ce qui a trait à ses opérations qu'à ses objectifs. Tous ces éléments peuvent ne pas se retrouver en même temps dans chaque entreprise.

1. Baumback et Mancuso (1975); Camara Oficial de comercio, Industria y navegacion de Barcelona (1989); Capiez (1988); Hudson (1989) ; Ikei (1987); Lafuente, Perez et Salas (1987); Veciana, 1989. 
L'étude de ces facteurs peut permette de caractériser chaque nouvelle entreprise et définir sa spécialisation, son niveau de diversification, sa situation géographique, sa capacité d'innovation, ses équipements de base et ses besoins réels.

La principale source de difficultés de ce type d'étude est le défaut de prise en compte des origines de l'entreprise. Par exemple, cette création peut résulter d'une réponse au manque de travail du promoteur plutôt que d'une préoccupation des besoins du marché. Sauf quelques exceptions notoires, ces nouvelles créations n'affectent pas fondamentalement le comportement du secteur correspondant.

Avant de discuter de ces questions, il faut remarquer que les typologies touchant les nouvelles entreprises ne sont pas très différentes de celles portant sur l'ensemble des $\mathrm{PME}^{2}$, ce que nous montrerons d'ailleurs en observant les résultats de notre recherche et en les comparant avec d'autres études.

Une réflexion sur les caractéristiques qui configurent le modèle retenu des nouvelles entreprises montre une étroite relation entre elles. Par exemple, le caractère individualiste des promoteurs explique un faible volume d'affaires de ces entreprises au démarrage et, ainsi, la faible importance économique de chaque unité. Les projets d'affaires naissants s'appuient habituellement plus sur les personnes physiques que sur les personnes juridiques, et lorsque parfois une activité rassemble plusieurs personnes, il y a toujours un nombre réduit d'associés, dépassant rarement trois ${ }^{3}$.

Cet individualisme des chefs d'entreprise explique aussi un certain conservatisme dans leur offre, conservatisme qui peut changer uniquement s'ils détectent des besoins demandant de plus grandes ressources. La recherche d'une certaine sécurité justifie des opérations peu ambitieuses et limite les attentes potentielles ${ }^{4}$. Il ne faut pas s'étonner qu'on ne retrouve que peu de firmes innovatrices ou d'idées originales qui méritent qu'on s'y arrête.

De la même façon, cette individualité implique un grand dévouement pour la cause avec, par conséquent, beaucoup de travail et de nombreuses responsabilités. Il semble paradoxal, compte tenu de cette situation, qu'on ne recoure pas plus à des aides externes qui soulageraient, physiquement et techniquement, ces charges et contribueraient à améliorer l'organisation souvent déficiente de ces firmes. Cette remarque est importante, surtout quand cette aide peut être ad hoc et peu coûteuse pour la firme.

2. Sur les typologies des PME et de leurs spécificités, on peut se référer à Auciello, Johnson et Wagenwald (1975) ou Hertz (1982).

3. Ceci peut s'expliquer par les limites fiscales et commerciales, comme l'expliquent Neila (1989) et Real Decreto Legislativo (1989).

4. Sur le profil des nouveaux chefs d'entreprise actuels, voir Claver et al. (1991). 
Tout projet de création provient d'une idée forte chez le promoteur. Quelques auteurs ${ }^{5}$ ont essayé de schématiser les options considérées lors de la période où se manifeste le souhait de monter une entreprise. Les chances de succès de cette exploration dépend généralement du degré de connaissance de l'environnement ou de la manière de l'envisager. La difficulté d'analyser toutes les possibilités de cet environnement ${ }^{6}$ ainsi que le caractère individualiste dont on a parlé plus haut conduisent le plus souvent l'entrepreneur à se limiter à un marché local, à se contenter de faibles ressources de départ et à se restreindre dans ses ambitions. Normalement, une bonne connaissance de cette idée de départ et de la façon dont elle a été structurée permet de mesurer les chances de viabilité de la nouvelle entreprise et de prévoir les principales difficultés à venir. Elle explique aussi les principales conditions pour développer adéquatement les opérations et pour organiser les moyens nécessaires. Elle peut aussi permettre de mieux définir les intentions des promoteurs et expliquer leur formation souvent insuffisante. En effet, cette formation est souvent très limitée, ce qui peut entraîner de fâcheuses conséquences.

Sur le plan opérationnel, une PME plus ancienne se différencie d'une nouvelle firme en cela qu'elle peut prévoir les solutions à ses problèmes, selon une certaine habitude. Cependant, dans les deux cas, des structures organisationnelles peu appropriées ou absentes pourront entraîner de graves difficultés.

Les problèmes provenant du manque d'expérience peuvent être atténués par la préparation d'un document formel, qui spécifie les principales étapes de la création, les diverses opérations et stratégies permettant le développement de l'entreprise, ainsi que les ressources humaines, physiques et financières nécessaires. Cet important outil interne de gestion, appelé plan d'affaire ${ }^{7}$, est fondamental dans la recherche d'appuis externes de toutes sortes. Malheureusement, la réalité montre qu'il est peu utilisé, ce qui s'explique en bonne partie par l'improvisation, le manque de préparation, l'excès de confiance, l'individualisme et le manque de vision de la part des nouveaux entrepreneurs.

Dans ce contexte, il n'est pas étonnant de voir que les jeunes propriétairesdirigeants ne modifient pas de façon notoire la structure de leur entreprise pour améliorer leur niveau de compétitivité. Cependant, ils peuvent évoluer si leur secteur industriel change. On retrouve dans ce cas les nouvelles entreprises

5. À ce sujet, voir Drucker (1985); Kuriloff et Hemphill (1986); Vesper (1980).

6. Toute publication sur la direction stratégique part du principe de la connaissance nécessaire des caractéristiques de l'environnement et de la complexité de cette tâche. On peut consulter Ansoff (1985); Grima Terre (1983); Porter (1987).

7. On peut approfondir la notion de «plan d'affaire» dans Mancuso (1983); Maqueda (1988); Rich et Gumpert (1985); Veciana (1988). 
considérées, selon la dénomination bien connues de Liles, (1974) comme des «entreprises marginales » qui, normalement, ne possèdent pas pour la plupart d'avantages particuliers par rapport aux autres entreprises. La singularisation des unes et des autres dépendra des conditions existantes dans chaque environnement particulier et de l'époque où elles apparaîtront.

\section{Application à la province d'Alicante}

Afin de voir si les caractéristiques générales dont nous venons de discuter existent dans la province d'Alicante, nous avons fait une enquête dans les entreprises créées depuis 1980. Notre premier travail fut de déterminer la population d'entreprises qui satisfaisait cette condition en utilisant les données de base provenant du registre de licences fiscales pour les entreprises. Ces données ont été scrutées pour éliminer toute erreur et fournir ainsi la base de données désirée.

De cette base, nous avons retenu un échantillon statistiquement représentatif. Nous avons ensuite divisé la population d'entreprises en strates homogènes nous permettant de faire des choix aléatoires partiels pour chaque strate. Les caractéristiques retenues sont: le type d'entreprise, la municipalité où elle est située et l'année de création. Le tout a été comparé aux mêmes strates de la population globale pour s'assurer de leur représentativité. Cela nous a donné 433 réponses valables, ce qui était statistiquement suffisant pour obtenir des conclusions fiables. Quant au questionnaire, qui a été testé auparavant, il a été conçu de façon à faire ressortir les raisons à la source de la création de chaque entreprise, raisons pouvant différer selon les localisations.

Nous avons ainsi pu mesurer l'importance des créations traditionnelles dans des activités «banales» des branches industrielles ou commerciales; nous avons trouvé que les petits commerces traditionnels étaient particulièrement nombreux, ce qu'avaient déjà noté les enquêtes de la Mairie ou de la Chambre de commerce de Barcelone, ou encore en France ${ }^{8}$, quant à la faible présence d'entreprises pouvant être qualifiées de technologiquement avancées, notamment dans le commerce au détail.

Majoritairement, l'origine des idées de départ des nouvelles entreprises est en forte relation entre le secteur où travaillait antérieurement l'entrepreneur et celui où évolue la nouvelle entreprise.

8. Camara Oficial de Comercio, Industria y navegacion de Barcelona (1989); Casabo (1989); Grau et Fil (1987). 
Au tableau 1, on voit aussi que la direction des nouvelles entreprises se limite à un seul, voire un maximum de trois administrateurs, ceci confirmant le caractère surtout individualiste des nouveaux entrepreneurs. Ce fait explique les faibles ressources de départ et l'importance des énergies mises en œuvre (un travail à plein temps), la grande responsabilité des nouveaux chefs d'entreprise.

\section{TABLEAU 1}

\section{Nombre d'associés au moment de la constitution}

\begin{tabular}{lcc}
\hline Nombre & Fréquence & $\%$ \\
\hline Un & 263 & 60,7 \\
Deux & 79 & 18,2 \\
Trois & 53 & 12,2 \\
Quatre & 19 & 4,4 \\
Cinq & 7 & 1,6 \\
Plus de cinq & 12 & 2,8 \\
\hline TOTAL & $\mathbf{4 3 3}$ & $\mathbf{1 0 0 , 0}$ \\
\hline
\end{tabular}

Comme il était à prévoir, la très grande majorité des nouvelles firmes (90,3\%), même après quelques années, se limite à un seul centre de production. De même, $84 \%$ d'entre elles ont moins de 5 employés et dans $40 \%$ des cas, aucun. Les employés ont le plus souvent une faible qualification. Bref, les nouvelles entreprises démarrent généralement toutes petites.

Sur le plan financier, les petites entreprises manquent de ressources à long terme. Elles ont des difficultés à accéder au marché de capitaux, n'ayant pas de garanties suffisantes pour amortir les risques ${ }^{9}$ (voir le graphique 1). Plus de la moitié des entrepreneurs (54\%) ont eu des difficultés à obtenir les capitaux nécessaires pour commencer leurs opérations et se développer convenablement.

Il est évident que l'obtention de capitaux de démarrage aura également un impact sur la taille finale de l'entreprise. Une étude espagnole (Lafuente et Yagüe (1989) a mesuré la relation entre le chiffre d'affaires de l'entreprise et sa structure financière en montrant que plus les entreprises sont petites, moins leur situation patrimoniale est appropriée. Le recours au crédit bancaire comme première source financière, parmi les différentes possibilités, est général.

9. Voir à ce propos, Sweeney (1987). 
GRAPHIQUE 1

\section{Difficulté dans l'obtention du financement externe}

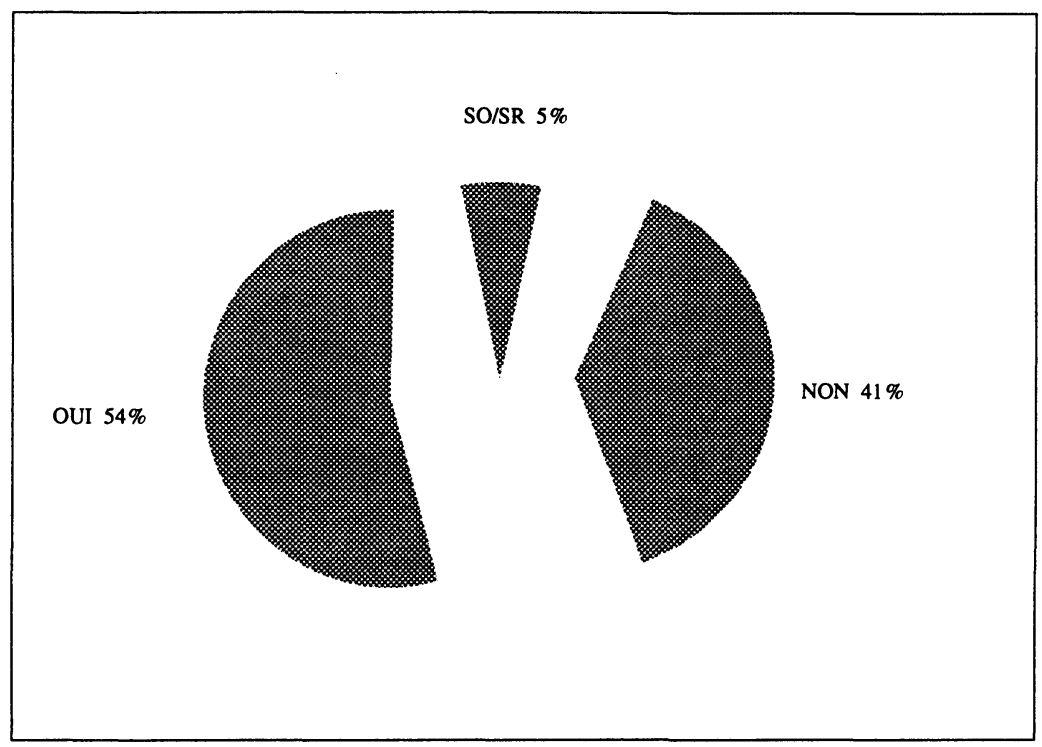

En abordant les moyens et les ressources utilisés lors de la création, nous avons demandé aux propriétaires-dirigeants comment ils considéraient le niveau technologique de leur entreprise. La majorité d'entre eux l'ont décrit comme «traditionnel» (tableau 2); ce qui explique l'état actuel de la technologie utilisée dans ces firmes et les risques que cette situation laisse entrevoir pour l'avenir.

TABLEAU 2

Nature de la technologie utilisée

\begin{tabular}{lcr}
\hline CAS & Fréquence & \% \\
\hline Traditionnelle & 259 & 59,8 \\
Avancée & 144 & 33,3 \\
SO/SR & 30 & 6,9 \\
\hline TOTAL & $\mathbf{4 3 3}$ & $\mathbf{1 0 0 , 0}$ \\
\hline
\end{tabular}

Si on observe la localisation du marché (graphique 2), on voit que celuici est avant tout local. En effet, 56,8\% des firmes se limitent au marché de leur localité et $75,7 \%$ des firmes ne vendent pas hors de la province. Ceci explique 
leur faible volume de ventes et leur centralisation dans un lieu unique de production, ce qui en revanche favorise leurs contacts directs avec les clients.

GRAPHIQUE 2

Domaine du marché

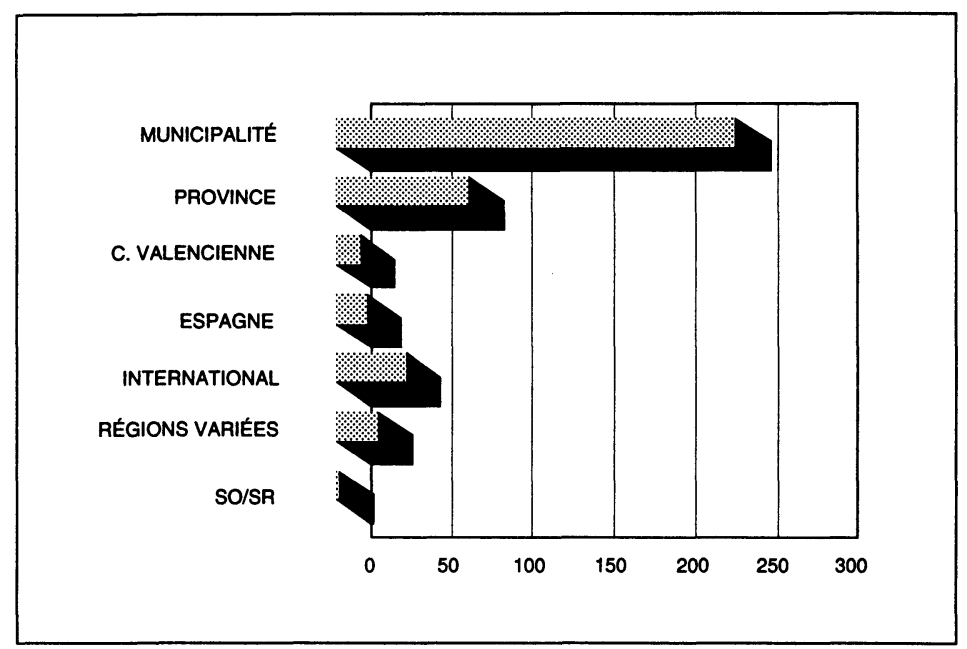

Du côté de la clientèle, nous remarquons d'abord son hétérogénéité. Seuls $7,6 \%$ des répondants affirment que leurs clients présentent les mêmes caractéristiques; ce qui laisse supposer que pour réaliser les ventes, les entrepreneurs doivent faire montre de flexibilité puisque leurs clients ont peu de comportements similaires, comme l'avaient déjà remarqué Fernandez et Oliva dans le cas des nouvelles entreprises ${ }^{10}$. Cette difficulté exigerait normalement plus d'innovation au plan de la commercialisation. Cependant, cette exigence semble ne pas être le cas chez $60 \%$ des répondants.

Quant à la prise de décisions, nous observons que dans $41,6 \%$ des cas, le chef d'entreprise décide tout seul, même pour une décision peu importante. Il consulte parfois ses collaborateurs, dans $35,3 \%$ des cas. Seules $23,1 \%$ des décisions sont prises avec les collaborateurs et ce, lorsqu'elles concernent leurs compétences particulières. Il serait souhaitable que, dans toute organisation, l'autorité soit mieux partagée afin de profiter des ressources existantes et favoriser leur engagement dans l'organisation (Maqueda, 1991).

10. Voir aussi Bueno Campo (1987). 
Du côté des infrastructures, on peut distinguer les principaux éléments suivants: la localisation et le coût des terrains et des locaux, l'approvisionnement en matériaux et en produits semi-finis, la technologie requise et le personnel approprié. Le tableau 3 montre clairement que le coût du terrain et des locaux est considéré comme le premier problème dans $44,3 \%$ des cas.

TABLEAU 3

Hiérarchisation des problèmes d'infrastructure

\begin{tabular}{|c|c|c|c|c|c|c|c|c|}
\hline \multirow[b]{2}{*}{ Hiérarchie } & \multicolumn{2}{|c|}{$\begin{array}{c}\text { Coût } \\
\text { des terrains } \\
\text { et des locaux }\end{array}$} & \multicolumn{2}{|c|}{$\begin{array}{l}\text { Approvisionnement } \\
\text { en matériaux } \\
\text { et produits }\end{array}$} & \multicolumn{2}{|c|}{$\begin{array}{l}\text { Technique } \\
\text { appropriée }\end{array}$} & \multicolumn{2}{|c|}{$\begin{array}{l}\text { Personnel } \\
\text { adéquat }\end{array}$} \\
\hline & $\mathbf{N}$ & $\%$ & $\mathbf{N}$ & $\%$ & $\mathbf{N}$ & $\%$ & $\mathbf{N}$ & $\%$ \\
\hline $1^{\text {er }}$ choix & 192 & 44,3 & 83 & 19,2 & 38 & 8,8 & 88 & 20,3 \\
\hline $2^{e}$ choix & 63 & 14,5 & 117 & 27,0 & 118 & 27,3 & 79 & 18,2 \\
\hline $3^{e}$ choix & 50 & 11,5 & 80 & 18,5 & 148 & 34,2 & 87 & 20,1 \\
\hline $4^{e}$ choix & 78 & 18,0 & 100 & 23,1 & 67 & 15,5 & 121 & 27,9 \\
\hline $\mathrm{SO} / \mathrm{SR}$ & 50 & 11,5 & 53 & 12,2 & 62 & 14,3 & 58 & 13,4 \\
\hline TOTAL & 433 & 100,0 & 433 & 100,0 & 433 & 100,0 & 433 & 100,0 \\
\hline
\end{tabular}

L'importance des problèmes légaux et financiers est illustrée au tableau 4. Ce sont d'abord les hausses des taux d'intérêts, suivi du poids des impôts, et ainsi leur incidence sur le financement, qui ressortent le plus. Si l'on retient aussi les deuxième, troisième et quatrième choix, l'inflation suivie des barrières législatives et la bureaucratie passent avant l'impôt et les taux d'intérêts.

TABLEAU 4

Hiérarchisation des problèmes légaux et financiers

\begin{tabular}{lrrrrrrrrr}
\hline & $\begin{array}{c}\text { Financement } \\
\text { et } \\
\text { intérêts }\end{array}$ & Impôts & Inflation & \multicolumn{2}{c}{$\begin{array}{c}\text { Législation } \\
\text { et } \\
\text { bureaucratie }\end{array}$} \\
\hline Hiérarchie & $\mathbf{N}$ & $\mathbf{\%}$ & $\mathbf{N}$ & $\mathbf{\%}$ & $\mathbf{N}$ & $\boldsymbol{\%}$ & $\mathbf{N}$ & $\%$ \\
$1^{\text {er choix }}$ & 152 & 35,1 & 149 & 34,4 & 24 & 5,5 & 93 & 21,5 \\
$2^{\text {e }}$ choix & 92 & 21,2 & 142 & 32,8 & 77 & 17,5 & 79 & 18,2 \\
$3^{\text {e }}$ choix & 54 & 12,5 & 84 & 19,4 & 117 & 27,0 & 98 & 22,6 \\
$4^{e}$ choix & 108 & 24,9 & 28 & 6,5 & 172 & 39,7 & 130 & 30,0 \\
SO/SR & 27 & 6,2 & 30 & 6,9 & 43 & 9,9 & 33 & 7,6 \\
\hline TOTAL & $\mathbf{4 3 3}$ & $\mathbf{1 0 0 , 0}$ & $\mathbf{4 3 3}$ & $\mathbf{1 0 0 , 0}$ & $\mathbf{4 3 3}$ & $\mathbf{1 0 0 , 0}$ & $\mathbf{4 3 3}$ & $\mathbf{1 0 0 , 0}$ \\
\hline
\end{tabular}


Sur l'aspect commercial, l'enquête montre que la concurrence déloyale et la difficulté d'augmenter et d'élargir les ventes viennent en premier et deuxième lieu (tableau 5). Les problèmes reliés aux intermédiaires ou aux distributeurs ne sont pas très importants, venant en troisième et quatrième position. Curieusement, et contrairement à ce que nous aurions pu penser étant donné les caractéristiques socio-économique de la province d'Alicante, le fait que le travail saisonnier soit très important, affectant ainsi les revenus et donc les ventes, n'a pas été souligné par les entrepreneurs.

TABLEAU 5

Hiérarchisation des problèmes commerciaux

\begin{tabular}{lrrrrrrrr}
\hline & $\begin{array}{c}\text { Concurrence } \\
\text { déloyale }\end{array}$ & \multicolumn{2}{c}{$\begin{array}{c}\text { Poids } \\
\text { de la concurrence } \\
\text { à court terme }\end{array}$} & $\begin{array}{c}\text { Difficulté } \\
\text { d'augmenter } \\
\text { les ventes }\end{array}$ & $\begin{array}{c}\text { Intermédiaires } \\
\text { et } \\
\text { distributeurs }\end{array}$ \\
\hline Hiérarchie & $\mathbf{N}$ & $\boldsymbol{\%}$ & $\mathbf{N}$ & $\mathbf{\%}$ & $\mathbf{N}$ & $\mathbf{\%}$ & $\mathbf{N}$ & $\mathbf{\%}$ \\
$1^{\text {er }}$ choix & 152 & 35,1 & 82 & 18,9 & 139 & 32,1 & 40 & 9,2 \\
$2^{\text {e }}$ choix & 92 & 21,2 & 109 & 25,2 & 105 & 24,2 & 95 & 21,9 \\
$3^{\text {e }}$ choix & 54 & 12,5 & 119 & 27,5 & 83 & 19,2 & 131 & 30,3 \\
$4^{\text {e }}$ choix & 108 & 24,9 & 83 & 19,2 & 74 & 17,1 & 127 & 29,3 \\
SO/SR & 27 & 6,2 & 40 & 9,2 & 32 & 7,4 & 40 & 9,2 \\
\hline TOTAL & $\mathbf{4 3 3}$ & $\mathbf{1 0 0 , 0}$ & $\mathbf{4 3 3}$ & $\mathbf{1 0 0 , 0}$ & $\mathbf{4 3 3}$ & $\mathbf{1 0 0 , 0}$ & $\mathbf{4 3 3}$ & $\mathbf{1 0 0 , 0}$ \\
\hline
\end{tabular}

Les nouveaux entrepreneurs ont, en général, une opinion passablement négative des gouvernements : $62,4 \%$ des répondants pensent que les normes gouvernementales entravent leur croissance, alors que $8,7 \%$ seulement estiment que les petites entreprises peuvent bénéficier de l'aide des pouvoirs publics (tableau 6) ${ }^{11}$. Ces résultats sont surprenants, compte tenu des différents besoins des nouvelles entreprises en aide externe (Mundet, 1991).

Bru et Jaime (1988) soulignent que les politiques industrielles mises en place depuis dix ans par la Communauté valencienne distinguent l'industrie traditionnelle et les nouvelles industries. Parmi les politiques qui favorisent ces dernières, on peut citer les «Centros de empresas e innovación» (CEI), le réseau des «Institutos tecnológicos sectoriales» et le «Parque tecnológico de Valencia». Ces institutions stimulent de différentes façons le développement de nouvelles entreprises locales. Cependant, le retard dans l'implantation effective

11. Signalons que dans les dernières années, l'aide provenant de la CEE a été particulièrement importante (Gamez et Ruiz, 1991 ; Ernst et Young, 1991). 
de ces mesures laisse présager que leurs répercussions ne se feront sentir qu'à moyen terme et n'affecteront pas de façon significative les chefs d'entreprise actuels.

\section{Tableau 6}

\section{Influences de l'État dans le développement d'entreprises}

\begin{tabular}{llr}
\hline & \multicolumn{1}{c}{ CAS } & $\%$ \\
\hline 1. & Les normes gouvernementales empêchent la croissance. & 62,4 \\
2. & Les conseils venant de l'Administration favorisent les PME. & 28,9 \\
3. Les petites entreprises bénéficient de l'aide de l'État. & 8,7 \\
4. & SO/SR & 0,0 \\
\hline & TOTAL & $\mathbf{1 0 0 , 0}$ \\
\hline
\end{tabular}

\section{Conclusion}

Le but de ce travail était d'étudier les caractéristiques générales des entreprises nouvelles, indépendamment de leur localisation, et de vérifier si les caractéristiques communes expliquant la création d'entreprise pouvaient se retrouver de façon relativement claire dans la province d'Alicante. La théorie explique que la plupart des nouvelles entreprises sont petites ; elles représentent individuellement un faible poids économique tant par le nombre d'associés que par le nombre d'employés et le volume d'opérations. Les entreprises d'Alicante sont conformes à cette image ; de plus, elles réalisent le plus souvent leurs opérations dans une seule unité de production.

Les problèmes que l'on y trouve ressemble à ceux relevés dans la documentation : elles ont des difficultés de financement et des problèmes d'infrastructure physique. Elles critiquent la fiscalité en place. Elles se plaignent de la concurrence déloyale, bien que cet aspect ne se retrouve pas dans toutes les entreprises. Par contre, l'inconvénient du travail saisonnier dans cette région et des fluctuations de revenus conséquents n'a pas été souligné, ce qui est étonnant, d'autant plus qu'on affirme que la clientèle est très hétérogène. Le marché demeure surtout local et la technologie employée est plutôt de type traditionnel.

Pour conclure, nous voulons revenir aux motivations à l'origine de la création des entreprises et ainsi à la difficulté de partir avec une culture commerciale forte. En effet, les créateurs d'entreprise, préoccupés par les problèmes concrets liés à cette création n'ont pas le temps de développer une vision et surtout de se rassembler pour développer des réseaux d'information leur permettant de 
mieux évaluer leur situation et de prévoir les changements à effectuer. Cependant, vu que les entreprises commencent habituellement toutes petites, elles sont plus à même de "sentir le vent», d'autant plus qu'elles sont bien impliquées dans leur milieu socioculturel, ce qui peut suppléer l'absence de liaisons avec des réseaux plus formels.

\section{Bibliographie}

ANSOFF, I. (1985), La dirección y su actitud frente al entorno, Bilbao, Deusto.

AuCIELlo, K.L., R. JohnSON et L.M. WAGENWALD (1975), An International Compilation of Small-Scale Industry Definitions, Cahier de recherche, Université d'État de Géorgie, É.-U.

BAUMBACK, C.M. et J. MANCUSO (1975), Entrepreneurship and Venture Management, Englewood Cliffs, New Jersey, Prentice-Hall.

BRU, S.Y. et V. JAIME (1988), «La Industria Valenciana: Balance de una década », Economía Industrial, $\mathrm{n}^{\circ}$ 263-264, sept.-déc., p. 281-294.

BUENO CAMPOS, E. (1987), La empresa española: estructura y resultados, Madrid, Instituto de Estudios Económicos.

Camara Oficial de Comercio, Industria y NAVEgacion de Barcelona (1989), L'emprenedor i la nova empresa, Barcelona.

CAPIEZ, A. (1988), «Conditions et modalités d'émergence des TPE et PE, l'exemple du Maine-et-Loire (France) », Revue Internationale P.M.E., vol. 1, n 2, p. 130-141.

CASABO, M. (1989), «Así son los nuevos emprendedores », Marketing y Ventas para Directivos, $\mathrm{n}^{\circ} 26$, mai, p. 32.

Claver, E. y otros (1991), Los nuevos empresarios alicantinos, Edita Fundesem, Banco de Alicante, CAM y CAPA.

DRUCKER, P.F. (1985), Innovation and Entrepreneurship, New York, Harper and Row.

ERNST et YOUNG (1991), Todo sobre ayudas e incentivos al sector industrial, Barcelona, Editorial Praxis.

FERNANDEZ, J. et M. OlIVA (1990), La creación de empresas: costes y problemática, Barcelona, Editorial Ariel, p. 87-101.

GRAU, R. et E. GIL (1987), Els nous empresaris a Barcelona, Ayuntamiento de Barcelona.

Grima TerRe, J.D. (1983), «Análisis de Entorno. Su nivel de introducción y sofisticación en la empresa española », Alta Dirección, n 110, p. 99-112. 
HERTZ, L. (1982), In Search of a Small Business Definition, Washington, University Press of America.

Hudson, J. (1989), "The Birth and Death of Firms», The Quarterly Review of Economics and Business, vol. 29, $\mathrm{n}^{\circ}$ 2, p. 68-86.

IKEI (1987), Dinamismo Empresarial del Pais Vasco en los años 80, Bilbao, Federación de Cajas de Ahorros Vasco-Navarras.

KuRILOFF, A.H. et J.M. HEMPHILl (1986), Cómo hacer rentable un negocio desde el principio. Factores básicos de eficacia, Bilbao, Deusto.

Lafuente, A., R. Perez et V. Salas (1987), «Creación de empresas y desarrollo económico: Evidencias internacionales», El Trimestre Económico, juilletseptembre, $\mathrm{n}^{\circ} 215$, p. $487-512$.

LAFUENTE, A. et M.J. YAGÜE (1989), «Estructura económica financiera de la empresa industrial española», Economía Industrial, mai-juin, ${ }^{\circ} 267$, p. 175-190.

LILES, P. (1974), New Business Ventures and the Entrepreneur, Homewood, Illinois, Richard D. Irwin.

Mancuso, J.R. (1983), How to Prepare a Business Plan, Englewood Cliffs, New Jersey, Prentice-Hall.

MAQUEDA, F.J. (1991), Creación y Dirección de Empresas, Barcelona, Editorial Ariel Economía, p. 151.

MUNDET, J. (1991), Creación de empresas: Factores de éxito, Barcelona, Editorial EINIA, p. 20-29.

NEILA NEILA, J.M. (1989), La nueva ley de sociedades anónimas, Madrid, Edersa, vol. I.

PORTER, M.E. (1987), Ventaja Competitiva, México, Cecsa.

Real DeCReto Legislativo 1.564/1.989, 22 décembre (BOE, 27 décembre 1989), p. $40012-40034$.

RICH, S.R. et D.E. GUMPERT (1985), Business Plans that Win $\$ \$$, New York, Harper and Row.

SWEENEY, G.P. (1987), Innovation, Entrepreneur and Regional Development, Londres, Frances Pinter P., p. 183.

Veciana, J.M. (1989), «Características del empresario en España », Papeles de Economía Española, $\mathrm{n}^{\circ}$ 39, p. 19-36.

VESPER, K.H. (1980), New Venture Strategies, Englewood Cliffs, New Jersey, PrenticeHall. 